\section{A microcomputer program for the analysis of interaction data}

\author{
JOHN WALKENBACH \\ University of Montana, Missoula, Montana 59812
}

Behavioral observation systems designed to allow the coding of naturally occurring interaction behaviors between people are becoming increasingly common. A detailed analysis of such behaviors is often very revealing. One method involves the formulation of several relevant and mutually exclusive behavior categories that maintain the identity of each of the participants. An observer then records the occurrence of these behavior categories at regular intervals (e.g., every $5 \mathrm{sec}$ ). This method preserves important information regarding antecedent-response and response-consequence relationships.

Data collected by this procedure are best displayed when they are reduced to a square matrix, the dimensions of which are equal to the number of behavioral categories. This may be done by pairing each of the $n$ observations (except for the final one) with the one following it, producing $n-1$ behavior code pairs. Each of these pairs is then tallied into the appropriate cell of the square interaction matrix. For example, one such pair might be 6-7 (Category 6 followed by Category 7). A tally would be made in Row 6 , Column 7 of the matrix. It then becomes apparent how many times a particular behavior followed (or preceded) any of the other behaviors. This information is useful in providing feedback to the participants and in designing specific interventions to change some aspects of the subjects' behaviors.

Perhaps one of the best known examples of this analytic procedure is the observation system developed by Flanders (Amidon \& Flanders, 1967) for use in analyzing classroom interactions occurring between a teacher and his or her students. The Flanders Interaction Analysis system has received a considerable amount of use in providing performance feedback to teachers (e.g., Amidon \& Flanders, 1967) and as a tool for classroom research (e.g., Williams, 1980).

The observation codes consist of 10 categories. Seven of the categories are for specific teacher behaviors, two are for student behaviors, and the final category is used to represent silence or confusion in the classroom. A detailed analysis of the observed sequences of these categories reliably indicates several aspects of classroom climate, teacher style, and teacher effectiveness.

An observation is made and the appropriate code is recorded every $3 \mathrm{sec}$ or when a category changes, which-

This work was supported in part by Grant HD14751-01 from the National Institute of Child Health and Human Development. ever occurs first. Thus, a large amount of data is rapidly accumulated. Transforming the sequence of observation codes into pairs and compiling the interaction matrix is only the beginning of a tedious task. Generally, the cells of the matrix are converted to percentages, column percentages are calculated, and several summary statistics are computed before the most useful information can be derived.

It is likely that this useful observation system is often neglected in favor of simpler but less informative procedures due to the time-consuming task of data reduction. One solution involves using a computer to perform the subsequent data reduction. The remainder of this paper describes such a program developed on the APPLE II microcomputer.

Program Description. The program interacts with the user, who selects one or more of the following functions: (1) to create a new data file on disk and receive input from the keyboard, (2) to edit or review on the screen an existing file, (3) to add additional data to an existing file, and (4) to create a matrix from the data contained in a file, calculate a number of summary statistics, and print the results.

The program is user oriented, and no knowledge of computers is needed beyond the ability to turn on the system and run the program. Input is facilitated by the use of GET statements; this allows reading keyboard input directly, without the need for a carriage return.

Output is in the form of a 10 by 10 matrix. Cell tallies and percents, as well as column totals and percents, are printed. In addition, the output includes the name of the input file, the number of observations, and summary statistics indicative of several aspects of teacher behavior. (See Amidon \& Flanders, 1967.)

Potential Modifications. Although the program was written specifically to analyze data collected from the Flanders coding system, modifications can be made to accommodate other observation systems that utilize the collection of a continuous sequence of discrete behavior codes. The format of the Flanders system has been incorporated into a number of widely used observation systems that are readily available (cf. Simon \& Boyer, 1974). We are currently developing a system that incorporates the Flanders interaction matrix concept to assess social interaction occurring between handicapped and nonhandicapped children in mainstreamed classrooms. Documentation within the program is sufficient to allow modifications to be made relatively easily.

Further program modifications could be made in the area of data input. For example, several portable solid state data collection devices on the market are capable of dumping data collected on site directly into a computer. The use of such a system would greatly facilitate using this program, since the data input would be performed automatically. 
Language and Hardware Requirements. The program is written in APPLESOFT BASIC and uses approximately $24 \mathrm{~K}$ bytes of memory. It is currently dimensioned to handle a maximum of 1,500 observations/file (approximately $75 \mathrm{~min}$ of 3 -sec interval recording). This may be altered by changing the DIM statement. A disk drive is needed for file storage. In addition, an 80column printer is necessary to accommodate the matrix printing format.

The only machine-dependent statements are those that perform the file manipulations. The program should run on other systems when the appropriate commands are submitted.

Availability. A listing of the program with sample input and output is available from J. Walkenbach,
Montana University Affiliated Program, University of Montana, Missoula, Montana 59812. Please include a large, self-addressed, stamped envelope.

\section{REFERENCES}

Amidon, E. J., \& Flanders, N. A. The role of the teacher in the classroom. Minneapolis: Association for Productive Thinking, 1967.

Srmon, A., \& Boyen, E. G. Mirrors for behavior III: An anthology of observation instruments. Wyncate, Penn: Communication Materials Center, 1974.

Williams, W. C. Pupil role and verbal "following behaviors." Education, 1980, 100, 326-335.

(Accepted for publication April 9, 1981.) 